\title{
Conductance enhancement in quantum-point-contact semiconductor-superconductor devices
}

Mortensen, Asger; Jauho, Antti-Pekka; Flensberg, Karsten; Schomerus, Henning

Published in:

Physical Review B

Link to article, DOI:

10.1103/PhysRevB.60.13762

Publication date:

1999

Document Version

Publisher's PDF, also known as Version of record

Link back to DTU Orbit

Citation $(A P A)$ :

Mortensen, A., Jauho, A-P., Flensberg, K., \& Schomerus, H. (1999). Conductance enhancement in quantumpoint-contact semiconductor-superconductor devices. Physical Review B, 60(19), 13762-13769.

https://doi.org/10.1103/PhysRevB.60.13762

\section{General rights}

Copyright and moral rights for the publications made accessible in the public portal are retained by the authors and/or other copyright owners and it is a condition of accessing publications that users recognise and abide by the legal requirements associated with these rights.

- Users may download and print one copy of any publication from the public portal for the purpose of private study or research.

- You may not further distribute the material or use it for any profit-making activity or commercial gain

- You may freely distribute the URL identifying the publication in the public portal 


\title{
Conductance enhancement in quantum-point-contact semiconductor-superconductor devices
}

\author{
Niels Asger Mortensen and Antti-Pekka Jauho \\ Mikroelektronik Centret, Technical University of Denmark, Ørsteds Plads, Building 345 East, DK-2800 Kgs. Lyngby, Denmark \\ Karsten Flensberg \\ Orsted Laboratory, Niels Bohr Institute, University of Copenhagen, Universitetsparken 5, DK-2100 Copenhagen $\emptyset$, Denmark \\ Henning Schomerus \\ Instituut-Lorentz, Universiteit Leiden, P.O. Box 9506, 2300 RA Leiden, The Netherlands
}

(Received 12 March 1999)

\begin{abstract}
We present numerical calculations of the conductance of an interface between a phase-coherent twodimensional electron gas and a superconductor with a quantum point contact in the normal region. Using a scattering matrix approach we reconsider the geometry of De Raedt, Michielsen, and Klapwijk [Phys. Rev. B 50, 631 (1994)] which was studied within the time-dependent Bogoliubov-de Gennes formalism. We find that the factor-of- 2 enhancement of the conductance $G_{\mathrm{NS}}$ compared to the normal state conductance $G_{\mathrm{N}}$ for ideal interfaces may be suppressed for interfaces with a quantum point contact with only a few propagating modes. The suppression is found to depend strongly on the position of the Fermi level. We also study the suppression due to a barrier at the interface and find an anomalous behavior caused by quasiparticle interference. Finally, we consider the limit of sequential tunneling and find a suppression of the factor-of-2 enhancement which may explain the absence of conductance enhancement in experiments on metal-superconductor structures.
\end{abstract}

[S0163-1829(99)07943-6]

\section{INTRODUCTION}

Charge transport through a normal-conductorsuperconductor (NS) interface is accompanied by a conversion of quasiparticle current to a supercurrent. In the Andreev reflection, by which the conversion occurs, an electronlike quasiparticle in the normal conductor (with an excitation energy lower than the energy gap of the superconductor) incident on the NS interface is retroreflected into a holelike quasiparticle (with reversal of its momentum and its energy relative to the Fermi level) and a Cooper pair is added to the condensate of the superconductor. ${ }^{1}$ For an ideal NS interface, the signature of Cooper pair transport and the Andreev scattering is a doubling of the conductance compared to the normal state conductance.

A theoretical framework for studies of the scattering at NS interfaces is provided by the Bogoliubov-de Gennes $(\mathrm{BdG})$ formalism, ${ }^{2}$ where the scattering states are eigenfunctions of the $\mathrm{BdG}$ equation

$$
\left(\begin{array}{cc}
\hat{\mathcal{H}}(\mathbf{r}) & \Delta(\mathbf{r}) \\
\Delta^{*}(\mathbf{r}) & -\hat{\mathcal{H}}^{*}(\mathbf{r})
\end{array}\right)\left(\begin{array}{l}
u(\mathbf{r}) \\
\mathrm{v}(\mathbf{r})
\end{array}\right)=E\left(\begin{array}{l}
u(\mathbf{r}) \\
\mathrm{v}(\mathbf{r})
\end{array}\right),
$$

which is a Schrödinger-like equation in electron-hole space (Nambu space). Here $\hat{\mathcal{H}}(\mathbf{r})$ is the single-particle Hamiltonian, $\Delta(\mathbf{r})$ is the pairing potential of the superconductor, $E$ is the excitation energy, and $u(\mathbf{r})$ and $\mathbf{v}(\mathbf{r})$ are wave functions of electronlike and holelike quasiparticles.

The technological possibility of studying the interface between a two-dimensional electron gas (2DEG) in a semiconductor heterostructure and a superconductor experimentally, has provided a playground for investigating the interplay be- tween the Andreev reflection and the mesoscopic effects seen in mesoscopic semiconductor structures. ${ }^{3,4}$ In recent years the technological efforts have revealed a variety of new mesoscopic phenomena, see Refs. 4-7, and references therein. One class of the studied devices are the quantum point contact (QPC) 2DEG-S and S-2DEG-S devices with the QPC in the normal region. The dc Josephson effect and the quantization of the critical current in QPC S-2DEG-S junctions have been studied extensively both experimentally by, e.g., Takayanagi and co-workers 8 and theoretically by, e.g., Beenakker and van Houten, ${ }^{9,10}$ Beenakker, ${ }^{11}$ and Furusaki, Takayanagi, and Tsukada. ${ }^{12}$

The linear-response conducting properties of QPC 2DEG-S structures have been studied by several groups. In the analytical work of Beenakker ${ }^{13}$ a ballistic normal region with a QPC modeled by a saddle-point potential was considered. The effect of elastic impurity scattering was considered numerically by Takagaki and Takayanagi ${ }^{14}$ who considered a disordered region between a narrow-wide (NW) constriction and the superconductor. Both of these studies of the conductance were based on a scattering matrix ( $S$-matrix) approach and the $\mathrm{BdG}$ formalism. In the numerical simulations of De Raedt, Michielsen, and Klapwijk, ${ }^{15}$ based on the timedependent BdG equation, a wide-narrow-wide (WNW) constriction was considered. Here, the aim was to study the electron-hole conversion efficiency and the robustness of the back-focusing phenomena of the Andreev reflection.

One of the properties of the QPC is that most transmission eigenvalues are either close to zero or unity. For an ideal QPC 2DEG-S interface, the Andreev reflection will therefore give rise to a factor-of-2 enhancement of the conductance $G_{\mathrm{NS}}$ compared to the normal state conductance $G_{\mathrm{N}},{ }^{10,13}$ which is quantized in units of $2 e^{2} / h .{ }^{16}$ However, as pointed 


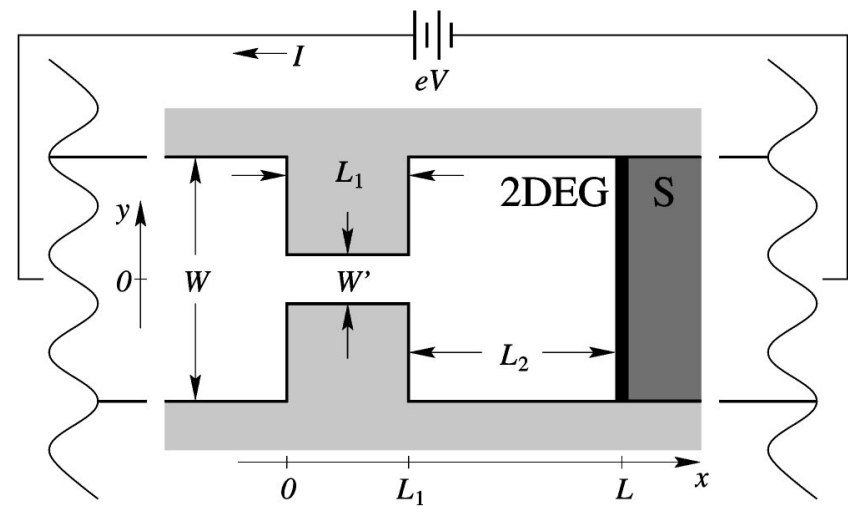

FIG. 1. Geometry of a WNW 2DEG-S junction with a hard-wall confining potential and a barrier at the 2DEG-S interface.

out by van Houten and Beenakker, ${ }^{10}$ deviations from the simple factor-of-2 enhancement should be expected when the position of the Fermi level does not correspond to a conductance plateau. The presence of impurity scattering in the normal region and/or interface roughness will also suppress the doubling of the conductance. ${ }^{14}$

Using an $S$-matrix approach, we study the linear-response regime of a phase-coherent ballistic QPC 2DEG-S system where the QPC is modeled by a WNW constrictions with a hard-wall confining potential, see Fig. 1. We report new results for the device studied by De Raedt et al. ${ }^{15}$ which had a relative width $W / W^{\prime}=1.7 \mu \mathrm{m} /(1.6 \times 335 \AA) \simeq 31.72$, an aspect ratio $L_{1} / W^{\prime}=5 / 1.6$, and a relative length $L_{2} / W^{\prime}$ $=20 / 1.6$. Applying the $S$-matrix formalism instead of the computationally more complicated time-dependent BdG formalism, we are able to study a larger part of the parameter space where we also consider a barrier (with a normalized barrier strength $Z$ ) at the NS interface. We focus on the regime with only a few propagating modes in the QPC. In this regime the transmission eigenvalues of the QPC depend strongly on the actual position of the Fermi level. Even for an ideal interface this gives rise to a strong suppression of the conductance for certain positions of the Fermi level as predicted by van Houten and Beenakker ${ }^{10}$ and subsequently seen in the work of Beenakker. ${ }^{13}$ In the presence of a barrier at the interface, the QPC gives rise to an enhanced tunneling through the barrier (compared to the case without a QPC) as in the case of reflectionless tunneling effect of diffusive junctions. ${ }^{13,17}$

In the sequential tunneling limit the conductance can be found by considering the QPC and the interface as two series-connected resistive regions and in the limit $W \gg W^{\prime}$ the enhancement of the conductance compared to the normal state conductance vanishes even for ideal NS interfaces. This may be an explanation for the unexpectedly low conductance enhancement in the experimental results of Benistant et al. ${ }^{18}$ on $\mathrm{Ag}-\mathrm{Pb}$ interfaces where the current is injected into the $\mathrm{Ag}$ crystal through a point contact.

The text is organized as follows. In Sec. II the $S$-matrix formalism is introduced, in Sec. III we formulate our model, in Sec. IV the scattering scheme of the considered geometry is presented, and in Sec. V we present results of several applications of our scattering scheme. Finally, in Sec. VI discussion and conclusions are given.

\section{SCATTERING MATRIX FORMALISM}

The scattering approach to coherent dc transport in superconducting hybrids follows closely the scattering theory developed for nonsuperconducting mesoscopic structures, see, e.g., the text-book by Datta. ${ }^{19}$ For an ideal NS interface, the interface acts as a phase-conjugating mirror within the Andreev approximation ${ }^{1}$ and the rigid boundary condition for the pairing potential

$$
\Delta(\mathbf{r})=\Delta_{0} e^{i \varphi} \Theta(x-L),
$$

where $\Delta_{0}$ is the BCS energy gap, ${ }^{20} \varphi$ is the phase of the pairing potential, $\Theta(x)$ is a Heaviside function, and $L=L_{1}$ $+L_{2}$ is the length of the normal region (see Fig. 1).

In the linear-response regime in zero magnetic field, Beenakker ${ }^{13}$ found that the conductance $G \equiv \partial I / \partial V$ is given by

$$
\begin{aligned}
G_{\mathrm{NS}} & =\frac{4 e^{2}}{h} \operatorname{Tr}\left(t t^{\dagger}\left[2 \hat{1}-t t^{\dagger}\right]^{-1}\right)^{2} \\
& =\frac{4 e^{2}}{h} \sum_{n=1}^{N} \frac{T_{n}^{2}}{\left(2-T_{n}\right)^{2}},
\end{aligned}
$$

which, in contrast to the Landauer formula, ${ }^{21}$

$$
G_{\mathrm{N}}=\frac{2 e^{2}}{h} \operatorname{Tr} t t^{\dagger}=\frac{2 e^{2}}{h} \sum_{n=1}^{N} T_{n},
$$

is a nonlinear function of the transmission eigenvalues $T_{n}$ $(n=1,2, \ldots, N)$ of $t t^{\dagger}$. Here $t$ is the $N \times N$ transmission matrix of the normal region, $N$ being the number of propagating modes.

Equation (3) holds for an arbitrary disorder potential and is a multichannel generalization of a conductance formula first obtained by Blonder, Tinkham, and Klapwijk ${ }^{22}$ who considered a delta function potential as a model for the interface barrier potential. The computational advantage of Eq. (3) over the time-dependent BdG approach of De Raedt et $a l^{15}$ is that we only need to consider the time-independent Schrödinger equation with a potential which describes the disorder in the normal region, so that we can use the techniques developed for quantum transport in normal conducting mesoscopic structures. For more details on, e.g., finite bias and/or temperature, see Lesovik, Fauchère, and Blatter, ${ }^{23}$ Lesovik and Blatter, ${ }^{24}$ and the reviews of Beenakker, ${ }^{6}$ and Lambert and Raimondi. ${ }^{7}$

\section{MODEL}

We describe the geometry of Fig. 1 by the Hamiltonian

$$
\hat{\mathcal{H}}(\mathbf{r})=-\frac{\hbar^{2}}{2 m} \hat{\nabla}+V_{\delta}(\mathbf{r})+V_{\mathrm{c}}(\mathbf{r})-\mu,
$$

where $\mu$ is the chemical potential. The barrier potential is given by a Dirac delta function potential with strength $H:^{22}$

$$
V_{\delta}(\mathbf{r})=H \delta(x-L),
$$

and the transverse motion is limited by a hard-wall confining potential 


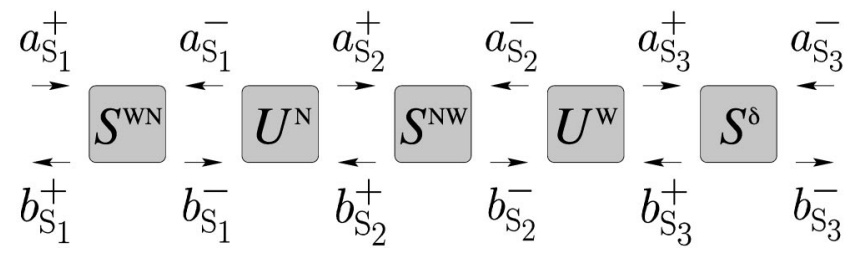

FIG. 2. Scattering scheme appropriate for the normal region of the geometry shown in Fig. 1.

$$
V_{\mathrm{c}}(\mathbf{r})=\left\{\begin{array}{cc}
0, & |y|<\mathcal{W}(x) / 2, \\
\infty, & |y| \geqslant \mathcal{W}(x) / 2,
\end{array}\right.
$$

where the width $\mathcal{W}(x)$ defines the WNW constriction and is given by

$$
\mathcal{W}(x)= \begin{cases}W, & x<0, \\ W^{\prime}, & 0 \leqslant x \leqslant L_{1}, \\ W, & x>L_{1} .\end{cases}
$$

The scattering states can be constructed as linear combinations of the eigenstates of the Schrödinger equation.

\section{SCATTERING SCHEME}

In the following subsections, we consider the $S$-matrices of a system with the Hamiltonian in Eq. (5) relevant for the geometry shown in Fig. 1. The $S$-matrix $S$ of a scattering region relates the incident current amplitudes $a_{\mathrm{S}}^{ \pm}$to the outgoing current amplitudes $b_{\mathrm{S}}^{ \pm}$. For a scattering region with two leads, $S$ is a $2 \times 2$ block-matrix with submatrices $S_{11}, S_{12}, S_{21}$, and $S_{22}$, where the diagonal and off-diagonal submatrices are reflection and transmission matrices, respectively. The appropriate scattering scheme for three scattering regions (the WN and the NW constrictions, and the interface barrier potential, respectively) connected by ballistic conductors is shown in Fig. 2.

The WN constriction is described by the $S$-matrix $S^{\mathrm{WN}}$

$$
\left(\begin{array}{c}
b_{\mathrm{S}_{1}}^{+} \\
b_{\mathrm{S}_{1}}^{-}
\end{array}\right)=S^{\mathrm{WN}}\left(\begin{array}{c}
a_{\mathrm{S}_{1}}^{+} \\
a_{\mathrm{S}_{1}}^{-}
\end{array}\right),
$$

the narrow region of length $L_{1}$ by the propagation-matrix $U^{\mathrm{N}}$

$$
\left(\begin{array}{c}
a_{\mathrm{S}_{1}}^{-} \\
a_{\mathrm{S}_{2}}^{+}
\end{array}\right)=U^{\mathrm{N}}\left(\begin{array}{c}
b_{\mathrm{S}_{1}}^{-} \\
b_{\mathrm{S}_{2}}^{+}
\end{array}\right)
$$

the NW constriction by the $S$-matrix $S^{\mathrm{NW}}$

$$
\left(\begin{array}{c}
b_{\mathrm{S}_{2}}^{+} \\
b_{\mathrm{S}_{2}}^{-}
\end{array}\right)=S^{\mathrm{NW}}\left(\begin{array}{c}
a_{\mathrm{S}_{2}}^{+} \\
a_{\mathrm{S}_{2}}^{-}
\end{array}\right),
$$

the wide region of length $L_{2}$ by the propagation-matrix $U^{\mathrm{W}}$

$$
\left(\begin{array}{c}
a_{\mathrm{S}_{2}}^{-} \\
a_{\mathrm{S}_{3}}^{+}
\end{array}\right)=U^{\mathrm{W}}\left(\begin{array}{c}
b_{\mathrm{S}_{2}}^{-} \\
b_{\mathrm{S}_{3}}^{+}
\end{array}\right)
$$

and the delta function barrier by the $S$-matrix $S^{\delta}$

$$
\left(\begin{array}{c}
b_{\mathrm{S}_{3}}^{+} \\
b_{\mathrm{S}_{3}}^{-}
\end{array}\right)=S^{\delta}\left(\begin{array}{c}
a_{\mathrm{S}_{3}}^{+} \\
a_{\mathrm{S}_{3}}^{-}
\end{array}\right) .
$$

To apply Eqs. (3) and (4) we need to calculate the composite transmission matrix $t=S_{21}$ which is a submatrix of the composite $S$-matrix $S \equiv S^{\mathrm{WN}} \otimes U^{\mathrm{N}} \otimes S^{\mathrm{NW}} \otimes U^{\mathrm{W}} \otimes S^{\delta}$ relating the outgoing current amplitudes to the incoming current amplitudes

$$
\left(\begin{array}{c}
b_{\mathrm{S}_{1}}^{+} \\
b_{\mathrm{S}_{3}}^{-}
\end{array}\right)=S\left(\begin{array}{c}
a_{\mathrm{S}_{3}}^{-} \\
a_{\mathrm{S}_{1}}^{+}
\end{array}\right) \quad S=\left(\begin{array}{ll}
r & t^{\prime} \\
t & r^{\prime}
\end{array}\right) .
$$

The meaning of the symbol $\otimes$ is found by eliminating the internal current amplitudes. ${ }^{19}$ As a final result we find the transmission matrix

$$
\begin{aligned}
t= & S_{21}^{\delta}\left[\hat{1}-U_{21}^{\mathrm{W}}\left\{S_{22}^{\mathrm{NW}}+S_{21}^{\mathrm{NW}}\left[\hat{1}-U_{21}^{\mathrm{N}} S_{22}^{\mathrm{WN}} U_{12}^{\mathrm{N}} S_{11}^{\mathrm{NW}}\right]^{-1} U_{21}^{\mathrm{N}} S_{22}^{\mathrm{WN}} U_{12}^{\mathrm{N}} S_{12}^{\mathrm{NW}}\right\} U_{12}^{\mathrm{W}} S_{11}^{\delta}\right]^{-1} \\
& \times U_{21}^{\mathrm{W}} S_{21}^{\mathrm{NW}}\left[\hat{\imath}-U_{21}^{\mathrm{N}} S_{22}^{\mathrm{WN}} U_{12}^{\mathrm{N}} S_{11}^{\mathrm{NW}}\right]^{-1} U_{21}^{\mathrm{N}} S_{21}^{\mathrm{WN}} .
\end{aligned}
$$

\section{A. Quantum point contact}

We consider a QPC which we model by a WNW constriction defined by a hard-wall confining potential, see Fig. 1. This geometry has been considered by Szafer and Stone ${ }^{25}$ and Weisshaar, Lary, Goodnick, and Tripathi ${ }^{26}$ in the context of conductance quantization of the QPC in a 2DEG, and recently by Kassubek, Stafford, and Grabert ${ }^{27}$ in the context of conducting and mechanical properties of ideal two and three-dimensional metallic nanowires. We follow Kassubek et $a .^{27}$ and calculate the composite $S$-matrix $S^{\mathrm{WNW}}=S^{\mathrm{WN}}$ $\otimes U^{\mathrm{N}} \otimes S^{\mathrm{NW}}$. In zero magnetic field, where all $S$-matrices satisfy $S=S^{T}$, the individual $S$-matrices are given by

$$
\begin{aligned}
S^{\mathrm{WN}} & =\left(\begin{array}{cc}
r_{\mathrm{NW}}^{\prime} & t_{\mathrm{NW}} \\
t_{\mathrm{NW}}^{T} & r_{\mathrm{NW}}
\end{array}\right), \\
U^{\mathrm{N}} & =\left(\begin{array}{cc}
\hat{0} & X^{\mathrm{N}} \\
X^{\mathrm{N}} & \hat{0}
\end{array}\right), \\
S^{\mathrm{NW}} & =\left(\begin{array}{ll}
r_{\mathrm{NW}} & t_{\mathrm{NW}}^{T} \\
t_{\mathrm{NW}} & r_{\mathrm{NW}}^{\prime}
\end{array}\right),
\end{aligned}
$$

where $X_{n n^{\prime}}^{\mathrm{N}}=\delta_{n n^{\prime}} \exp \left(i k_{n} L_{1}\right)$ describes the narrow region with free propagation of propagating modes and an exponen- 
tial decay of evanescent modes. Here $k_{n}$ $=k_{\mathrm{F}} \sqrt{1-\left(n \pi / k_{\mathrm{F}} W^{\prime}\right)^{2}}$ is the longitudinal wave vector of mode $n$ in the narrow region. The $S$-matrices of the $\mathrm{WN}$ and NW constrictions are related through an exchange of leads.

By elimination of the internal current amplitudes we find the composite transmission matrix

$$
S^{\mathrm{WNW}}=\left(\begin{array}{cc}
r_{\mathrm{WNW}} & t_{\mathrm{WNW}} \\
t_{\mathrm{WNW}} & r_{\mathrm{WNW}}
\end{array}\right),
$$

where

$$
\begin{gathered}
r_{\mathrm{WNW}}=r_{\mathrm{NW}}^{\prime}+t_{\mathrm{NW}}\left[\hat{1}-\left(X^{\mathrm{N}} r_{\mathrm{NW}}\right)^{2}\right]^{-1} X^{\mathrm{N}} r_{\mathrm{NW}} X^{\mathrm{N}} t_{\mathrm{NW}}^{T}, \\
t_{\mathrm{WNW}}=t_{\mathrm{NW}}\left[\hat{1}-\left(X^{\mathrm{N}} r_{\mathrm{NW}}\right)^{2}\right]^{-1} X^{\mathrm{N}} t_{\mathrm{NW}}^{T} .
\end{gathered}
$$

The $S$ matrix of the NW constriction can be found from a matching of scattering states which are eigenstates of the Schrödinger equation with the Hamiltonian in Eq. (5) where we only consider the part of the potential which sets up the NW constriction. From a matching of scattering states we find that

$$
\begin{gathered}
r_{\mathrm{NW}}=\left(\hat{1}+\varrho \varrho^{T}\right)^{-1}\left(\hat{1}-\varrho \varrho^{T}\right), \\
t_{\mathrm{NW}}=2 \varrho^{T}\left(\hat{1}+\varrho \varrho^{T}\right)^{-1}, \\
r_{\mathrm{NW}}^{\prime}=\left(\varrho^{T} \varrho+\hat{1}\right)^{-1}\left(\varrho^{T} \varrho-\hat{1}\right),
\end{gathered}
$$

where the elements of the $\varrho$ matrix can be written as $\varrho_{n w}$ $=\sqrt{K_{w} / k_{n}}\left\langle\phi_{n} \mid \Phi_{w}\right\rangle$, where $\left\langle\phi_{n} \mid \Phi_{w}\right\rangle=\int_{-\infty}^{\infty} \mathrm{d} y \phi_{n}(y) \Phi_{w}(y)$ is an overlap between transverse wave functions of mode $n$ in the narrow region and mode $w$ in the wide region. Here $K_{w}=k_{\mathrm{F}} \sqrt{1-\left(w \pi / \eta k_{\mathrm{F}} W^{\prime}\right)^{2}}$ is the longitudinal wave vector of mode $w$ in the wide region and $\eta \equiv W / W^{\prime}$ is the relative width of the constriction.

The overlap can be calculated analytically since its elements consist of overlaps between transverse wave functions $\phi_{n}$ and $\Phi_{w}$ which are either two sine or two cosine functions (the overlap between a sine function and a cosine function or vice versa is zero due to the odd and even character of the two functions). From the overlap-matrix we get the following elements of the $\varrho$ matrix:

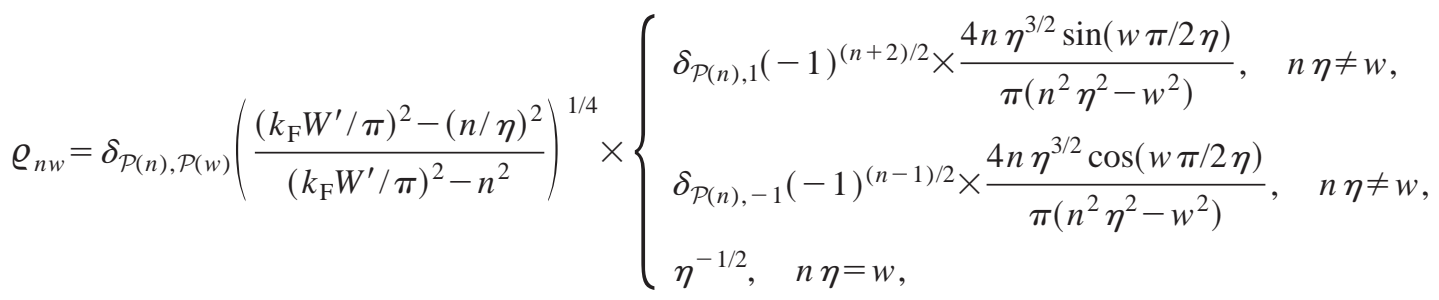

where the parity $\mathcal{P}(j)$ of $j$ is $\mathcal{P}(j) \equiv 1$ if $j$ is even and $\mathcal{P}(j)$ $\equiv-1$ if $j$ is odd.

In the numerical evaluation of Eqs. (22)-(24) and Eqs. (20) and (21) it is crucial to let the number of modes in the narrow and wide regions extend over both propagating modes and evanescent modes. After all matrix inversions are performed, the reflection and transmission matrices are projected onto the propagating modes. In practice, numerical convergence of the reflection and transmission matrices is found for a finite cutoff in the number of evanescent modes. For the considered device, the number of evanescent modes is roughly ten times the number of propagating modes in the wide region corresponding to $1000 \times 1000$ matrices.

In the limit $W \gg W^{\prime}$, Szafer and Stone ${ }^{25}$ employed a mean-field approximation for the overlap $\left\langle\phi_{n} \mid \Phi_{w}\right\rangle$ in which mode $n$ of the narrow region couples uniformly to only a band of modes (with the same parity as mode $n$ ) in the wide region within one level spacing so that the elements of the $\varrho$ matrix take the form

$$
\begin{aligned}
\varrho_{n w} \approx & \delta_{\mathcal{P}(n), \mathcal{P}(w)}\left(\frac{\left(k_{\mathrm{F}} W^{\prime} / \pi\right)^{2}-(n / \eta)^{2}}{\left(k_{\mathrm{F}} W^{\prime} / \pi\right)^{2}-n^{2}}\right)^{1 / 4} \\
& \times\left\{\begin{array}{l}
\eta^{-1 / 2}, \quad(n-1) \eta \leqslant w<(n+1) \eta, \\
0, \text { otherwise. }
\end{array}\right.
\end{aligned}
$$

Within this approximation, there is no mode mixing and $t t^{\dagger}$ becomes diagonal with the transmission eigenvalues along the diagonal. This approximation was found to capture the results of an exact numerical calculation ${ }^{25}$ when used with the Landauer formula, Eq. (4), which is linear in the transmission eigenvalues $T_{n}$. However, for an NS interface, the conductance formula, Eq. (3), is nonlinear in $T_{n}$ which also makes off-diagonal components in $t t^{\dagger}$ important. As we shall see (in Fig. 3), the mean-field approximation cannot reproduce the results of an exact numerical calculation of $G_{\mathrm{NS}}$ in the same nice way as for $G_{\mathrm{N}}$.

\section{B. Wide region}

The wide region $L_{1}<x<L$ is described similarly to the narrow region by

$$
U^{\mathrm{W}}=\left(\begin{array}{cc}
\hat{0} & X^{\mathrm{W}} \\
X^{\mathrm{W}} & \hat{0}
\end{array}\right),
$$

where $X_{w w^{\prime}}^{\mathrm{W}}=\delta_{w w^{\prime}} \exp \left(i K_{w} L_{2}\right)$ describes both the free propagating modes and the exponential decay of the evanescent modes. Here $K_{w}$ is the introduced longitudinal wave vector of mode $w$ in the wide region. 


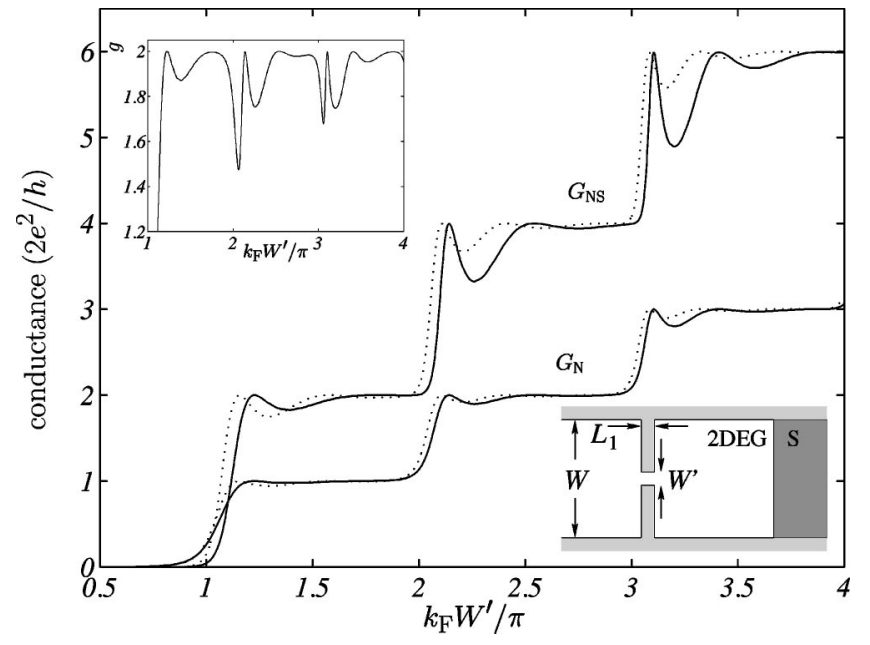

FIG. 3. Conductance $G_{\mathrm{NS}}$ and normal state conductance $G_{\mathrm{N}}$ of a coherent WNW 2DEG-S junction as a function of $k_{\mathrm{F}} W^{\prime} / \pi$. The constriction has an aspect ratio $L_{1} / W^{\prime}=1$ and a relative width $W / W^{\prime}=31.72$. The full lines are for a numerical calculation of $t_{\mathrm{WNW}}$ and the dotted lines are results within the mean-field approximation of Szafer and Stone (Ref. 25). The upper inset shows the normalized conductance $g \equiv G_{\mathrm{NS}} / G_{\mathrm{N}}$ also as function of $k_{\mathrm{F}} W^{\prime} / \pi$.

\section{Interface barrier potential}

We consider an NS interface of width $W$ with a barrier which we model by a Dirac delta function potential, following Blonder, Tinkham, and Klapwijk. ${ }^{22}$ The $S$-matrix elements for the delta-function potential is found from a matching of scattering states which are eigenstates of the Schrödinger equation with the Hamiltonian in Eq. (5) where we only consider the part of the potential consisting of the barrier at the interface. In zero magnetic field one finds the symmetric result

$$
S^{\delta}=\left(\begin{array}{ll}
r_{\delta} & t_{\delta} \\
t_{\delta} & r_{\delta}
\end{array}\right),
$$

with

$$
\begin{aligned}
& \left(t_{\delta}\right)_{w w^{\prime}}=\delta_{w w^{\prime}} \frac{1}{1+i Z / \cos \theta_{w}}, \\
& \left(r_{\delta}\right)_{w w^{\prime}}=\delta_{w w^{\prime}} \frac{-i Z / \cos \theta_{w}}{1+i Z / \cos \theta_{w}},
\end{aligned}
$$

where the normalized barrier strength is given by $Z$ $\equiv H / \hbar \mathrm{v}_{\mathrm{F}}$ and $\cos \theta_{w} \equiv K_{w} / k_{\mathrm{F}}=\sqrt{1-\left(w \pi / k_{\mathrm{F}} W\right)^{2}}$. The results differ from those of a one-dimensional calculation ${ }^{22}$ since we have taken the parallel degree of freedom into account. However, if we introduce an angle dependent effective barrier strength $Z_{\text {eff }}\left(\theta_{w}\right)=Z / \cos \theta_{w},{ }^{28,29}$ the transmission and reflection amplitudes can formally be written in the onedimensional form of Ref. 22. The transmission eigenvalues of $t_{\delta} t_{\delta}^{\dagger}$ are given by $T_{w}^{\delta}=\left[1+Z_{\mathrm{eff}}^{2}\left(\theta_{w}\right)\right]^{-1}$ in contrast to the mode-independent result $T_{w}^{\delta}=\left(1+Z^{2}\right)^{-1}$ of a onedimensional calculation. ${ }^{22}$

\section{RESULTS}

\section{A. Phase-coherent junction with ideal interface}

For the case of coherent transport through an ideal 2DEG-S interface with a WNW constriction in the normal region, see lower inset of Fig. 3, the conductance $G_{\mathrm{NS}}$ and the normal state conductance $G_{\mathrm{N}}$ can be found from Eqs. (3) and (4) with the transmission matrix $t=t_{\mathrm{WNW}}$. Fig. 3 shows the conductance as a function of $k_{\mathrm{F}} W^{\prime} / \pi$ based on a numerical calculation of $t_{\mathrm{WNW}}$ (full lines) and the mean-field approximation (dashed line) for a WNW constriction with an aspect ratio $L_{1} / W^{\prime}=1$ and a relative width $W / W^{\prime}=31.72$. The conductance $G_{\mathrm{NS}}$ is seen to be approximately quantized in units of $4 e^{2} / h$ which is twice the unit of conductance for the normal state conductance $G_{\mathrm{N}}$. However, just above the thresholds $\left(k_{\mathrm{F}} W^{\prime} / \pi=1,2,3, \ldots\right)$, oscillations due to resonances in the narrow region of the constriction are observed. In the normal state result, these resonances are small but in contrast to the Landauer formula, $G_{\mathrm{NS}}$ is not linear in the transmission eigenvalues and this makes the resonances much more pronounced compared to those in the normal state conductance. Another signature of the nonlinearity of $G_{\mathrm{NS}}$ and the importance of off-diagonal transmission, is that the mean-field approximation is in good agreement with the numerical calculation for $G_{\mathrm{N}}$ whereas it has difficulties in accounting for $G_{\mathrm{NS}}$. The sharpness of the resonances is to a certain extent due to the wide-narrow-wide constriction, and is suppressed in experiments with split-gate-defined constrictions. However, as shown by the simulations of Maaø, Zozulenko, and Hauge ${ }^{30}$ resonance effects do persist even for more smooth connections of the narrow region to the $2 \mathrm{DEG}$ reservoirs.

The normalized conductance $g \equiv G_{\mathrm{NS}} / G_{\mathrm{N}}$, shown in the upper insert, is two on the conductance plateaus but for certain "mode fillings" of the constriction it is strongly suppressed and for $k_{\mathrm{F}} W^{\prime} / \pi \sim 2$ (two propagating modes) we get $g \sim 1.5$. This effect, which occurs at the onset of new modes, was also seen in the calculations of Beenakker. ${ }^{13}$ As the number of modes increases, these dips vanish and the normalized conductance approaches its ideal value of 2 . The reason is simple: suppose the constriction has $N$ propagating modes, then the $N-1$ of them will have a transmission of order unity and only a single mode (corresponding to the mode with the highest transverse energy) will have transmission different from unity. As $N$ increases, the effect of the single mode with transmission different from unity becomes negligible for the normalized conductance and from Eqs. (3) and (4) it follows that $\lim _{N \rightarrow \infty} g=2$.

Since the quasiparticle propagation is coherent and the Andreev scattering is the only back-scattering mechanism, the phase conjugation between electronlike and holelike quasiparticles makes the conductance $G_{\mathrm{NS}}$ independent of the separation $L_{2}$ of the constriction and the interface. If evanescent modes in this region were also taken into account the results would depend weakly on $L_{2}$ as it was found in the simulations of De Raedt et al. ${ }^{15}$ and our results should be compared with their results in the large $L_{2}$ limit. As we shall see below, interfaces with a finite barrier (and thereby nor- 


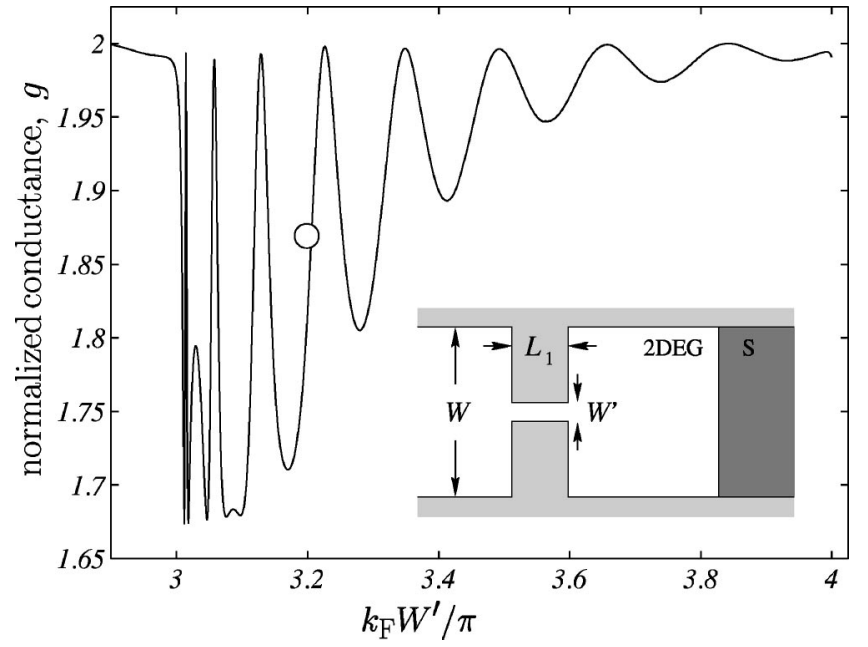

FIG. 4. Normalized conductance $g \equiv G_{\mathrm{NS}} / G_{\mathrm{N}}$ of a coherent WNW 2DEG-S junction as a function of $k_{\mathrm{F}} W^{\prime} / \pi$. The constriction has an aspect ratio $L_{1} / W^{\prime}=5 / 1.6$ and a relative width $W / W^{\prime}$ $=31.72$. The curve is based on a numerical calculation of $t_{\mathrm{WNW}}$. The data-point $(\bigcirc)$ corresponds to the numerical result $\left(k_{\mathrm{F}} W^{\prime} / \pi ; g\right)=(3.2 ; 1.87)$ of De Raedt et al. (Ref. 15, Table I).

mal scattering at the interface) lead to size quantization and thereby resonances which will depend on $L_{2}$.

The back-focusing phenomenon of the Andreev reflected quasiparticles and the lowering of the normalized conductance due to a QPC in the normal region was studied by De Raedt, Michielsen, and Klapwijk ${ }^{15}$ by solving the timedependent $\mathrm{BdG}$ equation fully numerically. In their wave propagation simulations, the QPC is also modeled by a WNW constriction with a relative width $W / W^{\prime}$ $=1.7 \mu \mathrm{m} /(1.6 \times 335 \AA) \simeq 31.72$, an aspect ratio $L_{1} / W^{\prime}$ $=5 / 1.6$, and a relative length $L_{2} / W^{\prime}=20 / 1.6$. For the particular " mode filling"' $k_{\mathrm{F}} W^{\prime} / \pi=3.2$, they find a normalized conductance $g=1.87<2$, but the dependence on the "mode filling" was not studied in detail.

In Fig. 4 we present a calculation of $g$ as a function of $k_{\mathrm{F}} W^{\prime} / \pi$ for this specific geometry. The result of De Raedt et al. $(\bigcirc)$ is reproduced but in general the normalized conductance is seen to have many resonances caused by the high aspect ratio of the constriction. In the range $3<k_{\mathrm{F}} W^{\prime} / \pi$ $<4$, the normalized conductance can be anything in the range $1.655<g \leqslant 2$ depending on the position of the Fermi level and though De Raedt et al. ${ }^{15}$ found the back-focusing phenomena of the Andreev reflection to be very robust with respect to changes of the device parameters, the normalized conductance itself certainly depends strongly on the position of the Fermi level. The reason is that only those quasiparticles which enter the region between the constriction and the interface can be Andreev reflected and thus contribute to the conductance enhancement compared to the normal state conductance.

\section{B. Phase-coherent junction with barrier at interface}

We next consider coherent transport through an NS interface with a barrier at the interface and a WNW constriction at a distance $L_{2}$ from the interface, see lower right inset of

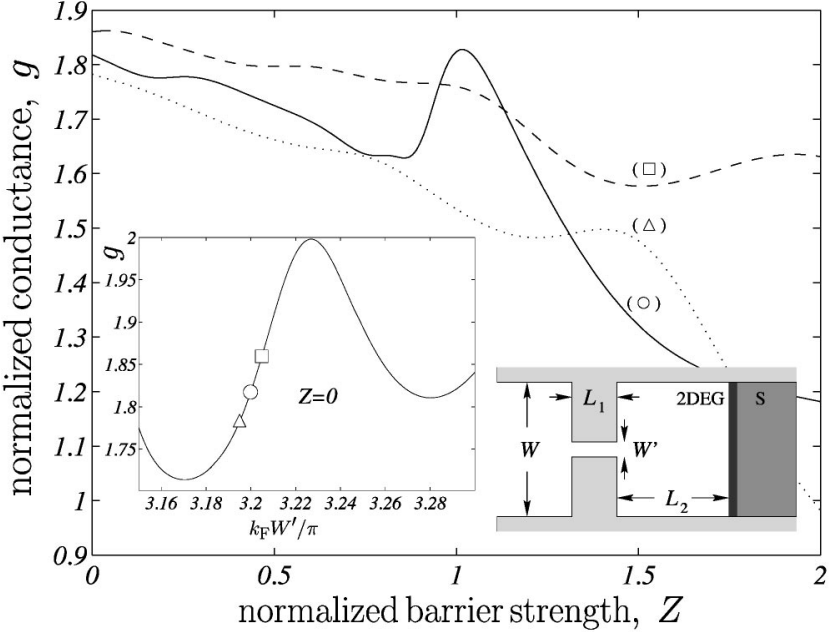

FIG. 5. Normalized conductance $g \equiv G_{\mathrm{NS}} / G_{\mathrm{N}}$ of a coherent WNW 2DEG-S junction with a barrier as a function of the normalized barrier strength $Z$ for $k_{\mathrm{F}} W^{\prime} / \pi=3.195(\triangle), k_{\mathrm{F}} W^{\prime} / \pi=3.2(\bigcirc)$, and $k_{\mathrm{F}} W^{\prime} / \pi=3.205(\square)$. The lower left inset shows the normalized conductance $g$ as a function of $k_{\mathrm{F}} W^{\prime} / \pi$ for $Z=0$. The constriction has an aspect ratio $L_{1} / W^{\prime}=5 / 1.6$, a relative width $W / W^{\prime}=31.72$, and the cavity has a relative length $L_{2} / W^{\prime}=20 / 1.6$.

Fig. 5. The conductance $G_{\mathrm{NS}}$ and the normal state conductance $G_{\mathrm{N}}$ are found from Eqs. (3) and (4) with the transmission matrix in Eq. (15).

In Fig. 5 we present a calculation of the normalized conductance $g$ as a function of the normalized barrier strength $Z$ for the device considered by De Raedt et al. ${ }^{15}$ For the position of the Fermi level ( $\bigcirc$ ) considered by De Raedt et al., the normalized conductance is only weakly suppressed (compared to a system without a QPC, see, e.g., Ref. 29) for low barrier scattering $(Z<1)$ and only for a very high barrier strength $(Z>2)$ the normalized conductance approaches the crossover from an excess conductance $(g>1)$ to a deficit conductance $(g<1)$. The effect of the barrier for $Z<1$ is very similar to the reflectionless tunneling behavior in diffusively disordered junctions ${ }^{13,17}$ where the net result is as if tunneling through the barrier is reflectionless. In the case of a QPC instead of a diffusive region there is a weak dependence on the barrier strength and the tunneling is not perfectly reflectionless.

An interesting feature is the nonmonotonic behavior of $g$ as a function of $Z$. For $Z \rightarrow \infty$, the normalized conductance of course vanishes, but in some regions it increases with an increasing barrier strength [curve $(O)]$ and for $Z \simeq 1$ it has the same value as for $Z=0$. This is purely an effect of size quantization in the cavity between the QPC and the barrier which enters the conducting properties because of the fully coherent propagation of electrons and holes. However, changing, e.g., the position of the Fermi level slightly [curves $(\triangle)$ and $(\square)]$, changes the quantitative behavior although the overall suppression of $g$ with increasing $Z$ is maintained.

\section{Incoherent junction}

In junctions where the propagation in the cavity between the QPC and the NS interface is incoherent, the so-called 


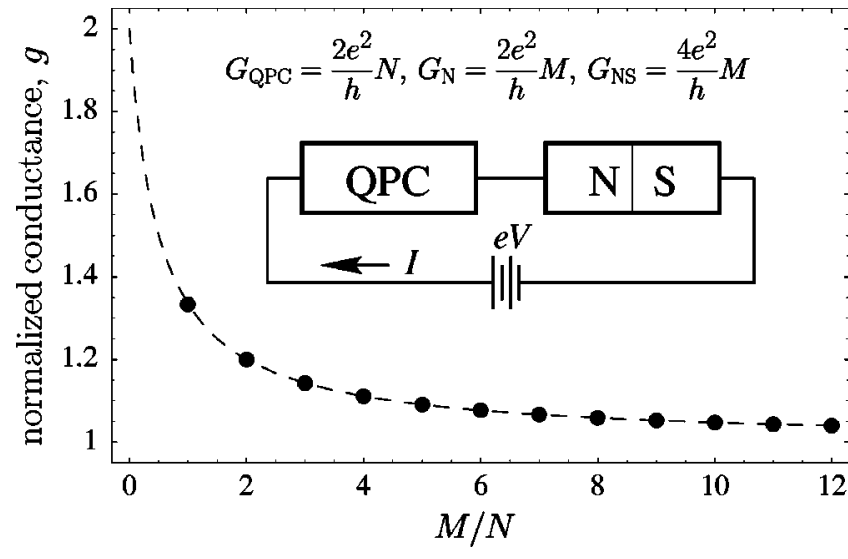

FIG. 6. Normalized conductance $g \equiv G_{\text {QPC-NS }} / G_{\text {QPC-N }}$ of a QPC NS junction with sequential tunneling through the ideal QPC and the ideal NS interface as a function of the ratio $M / N$ of propagating modes in the QPC and at the NS interface.

sequential tunneling regime, the QPC and the NS interface can be considered as two series-connected resistive regions. ${ }^{31}$ This means that

$$
\begin{gathered}
G_{\mathrm{QPC}-\mathrm{NS}}=\left(G_{\mathrm{QPC}}^{-1}+G_{\mathrm{NS}}^{-1}\right)^{-1}, \\
G_{\mathrm{QPC}-\mathrm{N}}=\left(G_{\mathrm{QPC}}^{-1}+G_{\mathrm{N}}^{-1}\right)^{-1},
\end{gathered}
$$

where $G_{\mathrm{QPC}}$ and $G_{\mathrm{N}}$ are found from Eq. (4) with $t=t_{\mathrm{QPC}}$ and $t=t_{\delta}$, respectively, and $G_{\mathrm{NS}}$ from Eq. (3) with $t=t_{\delta}$. The normalized conductance can be written as

$$
g \equiv \frac{G_{\mathrm{QPC}-\mathrm{NS}}}{G_{\mathrm{QPC}-\mathrm{N}}}=\frac{G_{\mathrm{NS}}}{G_{\mathrm{N}}} \times \frac{G_{\mathrm{QPC}}+G_{\mathrm{N}}}{G_{\mathrm{QPC}}+G_{\mathrm{NS}}},
$$

and for $W \gg W^{\prime}$ the major contribution to the resistance comes from the QPC, i.e., $G_{\mathrm{QPC}} \ll\left(G_{\mathrm{N}}, G_{\mathrm{NS}}\right)$. This means that the enhancement of $G_{\mathrm{NS}}$ compared to $G_{\mathrm{N}}$ has a negligible effect on the total conductance so that the normalized conductance approaches $g \sim 1$.

For an ideal QPC and an ideal interface we have $G_{\mathrm{QPC}}$ $=\left(2 e^{2} / h\right) N, G_{\mathrm{NS}}=\left(4 e^{2} / h\right) M$, and $G_{\mathrm{N}}=\left(2 e^{2} / h\right) M$, where $N$ is the number of modes in the QPC and $M$ is the number of modes at the NS interface. The corresponding normalized conductance is shown in Fig. 6.

The sequential tunneling behavior may provide an explanation for the unexpectedly small conductance enhancement seen in the experiments of Benistant et al. ${ }^{18}$ on Ag-Pb interfaces with injection of quasiparticles into an Ag crystal through a point contact. The condition for the electronic transport to be incoherent is that the distance between the point contact and the NS interface is longer than the correlation length $L_{c}=\operatorname{Min}\left(\ell_{\text {in }}, L_{\mathrm{T}}\right), \ell_{\text {in }}$ being the inelastic scattering length and $L_{T}$ the Thouless length. ${ }^{4,6}$ For the ballistic device studied by Benistant et al. $L_{c}=L_{T}=\hbar \mathrm{v}_{\mathrm{F}} / k_{\mathrm{B}} T$ $\sim 9 \mu \mathrm{m}$ (at $T=1.2 \mathrm{~K}$ ) which is much shorter than the distance between the point contact and the NS interface $(\sim 200 \mu \mathrm{m})$. Lowering the temperature will increase the correlation length and for sufficiently low temperatures $(T$ $\sim 0.05 \mathrm{~K})$ we expect a crossover from the sequential tunneling regime to the phase-coherent regime where the Andreev mediated conductance enhancement should become observable.

\section{DISCUSSION AND CONCLUSION}

For an ideal 2DEG-S interface with a QPC in the normal region, the normalized conductance $g \equiv G_{\mathrm{NS}} / G_{\mathrm{N}}$ depends strongly on the position of the Fermi level and only when the Fermi level corresponds to a conductance plateau a doubling of the conductance is found. The deviations from the factorof- 2 enhancement, when the Fermi level does not correspond to a plateau, can be significant and for a particular example of the WNW constriction we find that the normalized conductance can be suppressed to $g \sim 1.5$ in a system with only two propagating modes in the constriction. In the presence of a barrier at the 2DEG-S interface, the normalized conductance depends strongly on the longitudinal quantization in the cavity set up by the QPC and the barrier. Depending on the barrier strength, the length of the cavity and the position of the Fermi level, this longitudinal quantization may give rise to both constructive and destructive inferences in the transmission and thus also in the conductance. Perhaps surprisingly, the effect of the barrier is very much suppressed (compared to a system without a QPC, see, e.g., Ref. 29) due to a very strong back scattering at the return of the quasiparticles to the normal probe. The localization of quasiparticles in the cavity gives rise to an almost reflectionless tunneling through the barrier as it is also found in systems with a diffusive normal region. ${ }^{13,17}$ The interferences due to localization in the cavity will be smeared by a finite temperature and they are also expected to be suppressed by a finite inelastic scattering length compared to the length of the cavity. $^{32}$

For the sequential tunneling regime we find that the conductance enhancement vanishes as the number of modes at the interface becomes much larger than the number of modes in the QPC. Our calculations show that the $S$-matrix approach provides a powerful alternative to the time-dependent Bogoliubov-de Gennes approach of De Raedt et al. ${ }^{15}$ in detailed studies of the conducting properties of nanoscale 2DEG-S devices. Even though the back-focusing phenomenon of the Andreev reflection is robust against changes in the geometry, ${ }^{15}$ the electron-hole conversion efficiency itself is not. Finally, we stress that for a quantitative comparison to experimental systems, it is crucial to take different Fermi wave vectors and effective masses of the 2DEG and the superconductor into account. ${ }^{29}$

\section{ACKNOWLEDGMENTS}

We would like to thank C.W.J. Beenakker, M. Brandbyge, J.B. Hansen, and H.M. Rønnow for useful discussions. N.A.M. acknowledges financial support by the Nordic Academy for Advanced Study (NorFA) and H.S. acknowledges support by the European Community. 
${ }^{1}$ A.F. Andreev, Zh. Éksp. Teor. Fiz. 46, 1823 (1964) [Sov. Phys. JETP 19, 1228 (1964)].

${ }^{2}$ P.G. de Gennes, Superconductivity of Metals and Alloys (Benjamin, New York, 1966).

${ }^{3}$ C.W.J. Beenakker and H. van Houten, Solid State Phys. 44, 1 (1991).

${ }^{4}$ Y. Imry, Introduction to Mesoscopic Physics (Oxford University Press, Oxford, 1997).

${ }^{5}$ T.M. Klapwijk, Physica B 197, 481 (1994).

${ }^{6}$ C.W.J. Beenakker, in Mesoscopic Quantum Physics, edited by E. Akkermans, G. Montambaux, and J.-L. Pichard (North-Holland, Amsterdam, 1995); Rev. Mod. Phys. 69, 731 (1997).

${ }^{7}$ C.J. Lambert and R. Raimondi, J. Phys.: Condens. Matter 10, 901 (1998).

${ }^{8}$ H. Takayanagi, J.B. Hansen, and J. Nitta, Phys. Rev. Lett. 74, 166 (1995); H. Takayanagi, T. Akazaki, and J. Nitta, ibid. 75, 3533 (1995); H. Takayanagi and T. Akazaki, Phys. Rev. B 52, R8633 (1995); H. Takayanagi, Phys. Rev. B 218, 113 (1996); H. Takayanagi, ibid. 227, 224 (1996); H. Takayanagi, T. Akazaki, and J. Nitta, Surf. Sci. 362, 298 (1996); H. Takayanagi, T. Akazaki, and E. Toyoda, Appl. Supercond. 5, 357 (1997).

${ }^{9}$ C.W.J. Beenakker and H. van Houten, Phys. Rev. Lett. 66, 3056 (1991).

${ }^{10}$ H. van Houten and C.W.J. Beenakker, Physica B 175, 187 (1991).

${ }^{11}$ C.W.J. Beenakker, Phys. Rev. Lett. 67, 3836 (1991); 68, 1442 (1992).

${ }^{12}$ A. Furusaki, H. Takayanagi, and M. Tsukada, Phys. Rev. Lett. 67, 132 (1991); Physica C 185-189, 2585 (1991); Phys. Rev. B 45, 10563 (1992).

${ }^{13}$ C.W.J. Beenakker, Phys. Rev. B 46, 12841 (1992).

${ }^{14}$ Y. Takagaki and H. Takayanagi, Phys. Rev. B 53, 14530 (1996).

${ }^{15}$ H. De Raedt, K. Michielsen, and T.M. Klapwijk, Phys. Rev. B 50, 631 (1994)

${ }^{16}$ B.J. van Wees, H. van Houten, C.W.J. Beenakker, J.G. Williamson, L.P. Kouwenhoven, D. van der Marel, and C.T. Foxon,
Phys. Rev. Lett. 60, 848 (1988); D.A. Wharam, T.J. Thornton, R. Newbury, M. Pepper, H. Ahmed, J.E.F. Frost, D.G. Hasko, D.C. Peacock, D.A. Ritchie, and G.A.C. Jones, J. Phys. C 21, L209 (1988).

${ }^{17}$ B.J. van Wees, P. de Vries, P. Magnée, and T.M. Klapwijk, Phys. Rev. Lett. 69, 510 (1992).

${ }^{18}$ P.A.M. Benistant, A.P. van Gelder, H. van Kempen, and P. Wyder, Phys. Rev. B 32, 3351 (1985).

${ }^{19} \mathrm{~S}$. Datta, Electronic Transport in Mesoscopic Systems (Cambridge University Press, Cambridge, 1995).

${ }^{20}$ J. Bardeen, L.N. Cooper, and J.R. Schrieffer, Phys. Rev. 108, 1175 (1957).

${ }^{21}$ R. Landauer, IBM J. Res. Dev. 1, 223 (1957); Philos. Mag. 21, 863 (1970); D.S. Fisher and P.A. Lee, Phys. Rev. B 23, 6851 (1981); M. Büttiker, Phys. Rev. Lett. 57, 1761 (1986).

${ }^{22}$ G.E. Blonder, M. Tinkham, and T.M. Klapwijk, Phys. Rev. B 25, 4515 (1982).

${ }^{23}$ G.B. Lesovik, A.L. Fauchère, and G. Blatter, Phys. Rev. B 55, 3146 (1997).

${ }^{24}$ G.B. Lesovik and G. Blatter, Pis'ma Zh. Eksp. Teor. Fiz. 68, 572 (1998) [JETP Lett. 68, 599 (1998)].

${ }^{25}$ A. Szafer and A.D. Stone, Phys. Rev. Lett. 62, 300 (1989).

${ }^{26}$ A. Weisshaar, J. Lary, S.M. Goodnick, and V.K. Tripathi, J. Appl. Phys. 70, 355 (1991).

${ }^{27}$ F. Kassubek, C.A. Stafford, and H. Grabert, Phys. Rev. B 59, 7560 (1999).

${ }^{28}$ M. Kupka, Physica C 281, 91 (1997).

${ }^{29}$ N.A. Mortensen, K. Flensberg, and A.-P. Jauho, Phys. Rev. B 59, 10176 (1999).

${ }^{30}$ F.A. Maaø, I.V. Zozulenko, and E.H. Hauge, Phys. Rev. B 50, 17 320 (1994).

${ }^{31}$ M. Büttiker, Phys. Rev. B 33, 3020 (1986); IBM J. Res. Dev. 32, 72 (1988).

${ }^{32}$ For a recent approach including possible interactions, see, e.g., Q. Sun, J. Wang, and T. Lin, Phys. Rev. B 59, 3831 (1999). 